\title{
Changes of Reporting Rates in the Southern California Earthquake Catalog, Introduced by a New Definition of $M_{\mathrm{L}}$
}

\author{
by Thessa Tormann, Stefan Wiemer, and Egill Hauksson
}

\begin{abstract}
Starting January 2008, local magnitudes $M_{\mathrm{L}}$ for southern California are determined by a new calibration that provides various improvements for determining $M_{\mathrm{L}}$ for small earthquakes. Magnitudes for the previous years are being recalculated and the catalog continuously updated, with the first year of overlapping data now being available. Recalibrating a magnitude scale can cause a break in homogeneity of reporting and often produces artifacts in the catalog statistics that can influence a wide range of seismicity studies. To search for such a break, we compare the old $M_{\mathrm{L}}$ and the new $M_{\mathrm{L}}$ catalogs for 2007. We find (1) the two magnitude values differ for $96 \%$ of the $M_{\mathrm{L}}$ events, and hand-determined magnitudes are also revised; (2) the magnitude differences are irregular from magnitude increases of up to 1.5 units to reductions by as much as 2.3 units, with an average change of -0.13 units; (3) the number of events above $M 1.8$ decreases by $32 \%$ for the new magnitude scale; (4) the completeness magnitude apparently drops by 0.3 units from 1.6 to 1.3 ; $(5)$ the $b$-value reduces by approximately 0.2 units, dropping from 1.16 to 0.95 ; (6) the new magnitude calibration produces a more stable $b$-value estimate and can therefore be regarded as the better scaling.

We document selected examples of how the change in magnitude calibration may affect seismicity- and hazard-related analyses that are based on the Southern California Seismic Network (SCSN) catalog. Especially the change of the $b$-value from $\sim 1.1$ to $\sim 0.9$ has potentially major implications for hazard related applications.
\end{abstract}

\section{Introduction}

In January 2008 the California Integrated Seismic Network (CISN) introduced a new method to calculate local magnitudes, $M_{\mathrm{L}}$, in southern California. CISN therewith eliminated the heterogeneity in $M_{\mathrm{L}}$ definitions within the State of California. Since the local magnitude scale was developed by Richter in 1935 (Richter, 1935), not only have several different $M_{\mathrm{L}}$ definitions been used for different places in California, but they have also often been adapted when new stations were added to the networks. For the recalibration, earthquake data from all of California were analyzed and reconvolved into Wood-Anderson seismograph responses to calculate synthetic Wood-Anderson amplitudes. The resulting more than five million observations of station-network-channel-location differences were simultaneously inverted to determine a new statewide-valid attenuation relation as well as new site corrections for all stations. Constraints were applied in an attempt to ensure consistency with past magnitudes in northern and southern California (Kanamori et al., 1999; Hellweg et al., 2007; Uhrhammer and Hellweg et al., unpublished manuscript, 2009). Further, for small earthquakes, a high-pass filter is applied to the waveforms to remove ocean microseisms that may affect the amplitude values. The new procedure is expected to provide more accurate and more robust magnitudes, especially for $M<5.0$ events throughout all of California. The Southern California Seismic Network (SCSN) catalog that is distributed by the Southern California Earthquake Data Center (SCEDC) lists the new magnitudes from 1 January 2008 onward. The widely used Advanced National Seismic System (ANSS) catalog, which compiles reports from a variety of regional and global networks, also reports the new magnitudes for southern California.

In May 2009 the SCSN started to recalculate $M_{\mathrm{L}}$ values back to 1992 or to the time when the operation of the original Wood-Anderson seismometers was discontinued and broadband records became available for generating synthetic Wood-Anderson records. The recalculation of these past magnitudes will take several years. By now, the first year of overlapping data, 2007, has become available, offering the excellent opportunity to thoroughly analyze the impacts of the change in $M_{\mathrm{L}}$ calibration on the homogeneity of reporting in the southern California catalog.

Southern California is the region with the highest seismic risk in the United States, owing to the high seismicity 
rate and the large population centers. Consequently, southern California has been for many decades one of the seismically best monitored and best studied regions in the world. The SCSN is a complex seismic network, recording data from more than 400 stations with different types of sensors and with different local geotechnical site characteristics. Many of the procedures used routinely today in seismology have originated here, including, of course, the local magnitude scale introduced by Richter in 1935 (Richter, 1935), as well as the commonly used description of the frequency of earthquakes

$$
\log (N)=a-b M,
$$

defined like that by Gutenberg and Richter in the 1940s (Gutenberg and Richter, 1944). The earthquake catalog of southern California has been used in hundreds, if not thousands, of publications related to earthquake physics, statistical seismology, and seismic hazard. The catalog has been thoroughly analyzed for its completeness level (e.g., Wiemer and Wyss, 2000; Woessner and Wiemer, 2005; Schorlemmer and Woessner, 2008) and homogeneity in space and time.

Network operators worldwide strive to improve their network, where improvement for the purpose of earthquake catalog creation is generally measured by the ability to detect smaller events (a decrease in the magnitude of completeness, $M_{c}$ ) or the ability to locate events more precisely. This can be achieved, for example, by adding more stations, improving the signal to noise characteristic of stations, or by improving the subsequent signal processing (e.g., filtering, triggering, magnitude calculation). Unfortunate consequences of these improvements are changes in the homogeneity and consistency of reporting. Those artificial (or man-made) changes in reporting, such as apparent changes in rate, changes in $M_{c}$ and $b$-values (eq. 1), or shifts or stretches of the whole magnitude scale, are common in all earthquake catalogs (Habermann, 1987; Habermann and Craig, 1988; Zuniga and Wiemer, 1999).

Such artifacts pose a challenge for seismological research because they can mimic or mask natural changes. Examples include, but are by no means limited to: earthquake triggering, precursory signals, and spatial and temporal mapping of seismicity parameters such as $b$-values. Changes in the homogeneity of reporting can also alter estimated seismicity parameters, which then alters the seismic hazard and risk assessment for a region. Finally, man-made changes have the potential to bias prospective testing of earthquake forecasting, for example, in the framework of the Collaboratory for the Study of Earthquake Predictability (CSEP) (Schorlemmer et al., 2010).

The change in data processing introduced by the SCSN in January 2008 is a prime example of a change in reporting homogeneity. While the new procedures improve the consistency of the scaling of $M_{\mathrm{L}}$, they also introduce, as we document here, significant changes in the reporting rate of microseismicity and the apparent relative earthquake-size distribution. The SCSN $M_{\mathrm{L}}$ recalibration is an excellent case study for the kind of challenges networks and researchers face. Many other networks in the coming years will introduce similar site corrections, for example, the Northern California Seismic Network, which is changing to the same new $M_{\mathrm{L}}$ scale at the time of this writing; this article offers insight into the kinds of changes that will occur.

\section{Data}

We use two earthquake catalogs: the current SCSN catalog available from their website (giving the old $M_{\mathrm{L}}$ calibration values up to the end of 2006 and the new values starting January 2007 [status of September 2009]) and the SCSN catalog for 2007 that we downloaded in early January 2008, giving the old $M_{\mathrm{L}}$ values. We have chosen both catalogs to contain local events only (i.e., they are dequarried), and include events from all depths and with magnitudes $M>0$. Our analysis concentrates on the 2007 data, for which values from both $M_{\mathrm{L}}$ calibrations are available. For part of the analysis we need a longer catalog period, covering 2002 to 2008.

To ensure highest data quality, we define our study region to be the polygon of $M_{c}$ equals 1.8 completeness inside the rectangular region between $32^{\circ}$ and $37^{\circ}$ latitude and $-121^{\circ}$ and $-114.5^{\circ}$ longitude, as determined by the probability-based magnitude of completeness (PMC) method (Schorlemmer and Woessner, 2008) at a probability level of 0.999 (Fig. 1a). The PMC method computes a network's completeness map at a specific time by using empirical data only: the earthquake catalog, a station list with on and off times, and the magnitude definition used by the network. From this information it compiles, for each station, a matrix indicating the probability of detecting an earthquake of certain magnitude and distance. The advantage of this completeness level approach is that it does not assume linear behavior of the frequency magnitude distribution (FMD), as done, for example, in the entire magnitude range (EMR) method (Woessner and Wiemer, 2005). The latter approach is modeling both the complete and incomplete part of the FMD, returning a maximum-likelihood estimate of $M_{c}$. The results proved to be superior to estimates from cumulative FMDfitting methods; therefore, EMR is taken as the traditional alternative to the PMC values in this study. Taking the PMC value to be the minimum magnitude of complete recording is a rather conservative assumption, because traditional completeness estimation methods, for example, the EMR algorithm, suggest values between 1.3 (for new $M_{\mathrm{L}}$ data) and 1.6 (for old $M_{\mathrm{L}}$ data) for this region.

The resulting SCSN catalog for 2007 contains 10,104 events for which we have both magnitude values; maximum magnitude is $M$ 4.7. The longer period from 2002 to 2008 contains 74,533 events with maximum magnitude $M_{\mathrm{w}} 5.4$ (Fig. 1a). Before the recalibration (the following numbers based on the catalog for 2002 to 2006), about $75 \%$ of the events are assigned a local magnitude $M_{\mathrm{L}}$, less than half a percent splits between $M_{\text {coda }}$ (latest annotations in 2003) and very few moment magnitudes $M_{\mathrm{w}}$, and nearly $25 \%$ are 
(a)

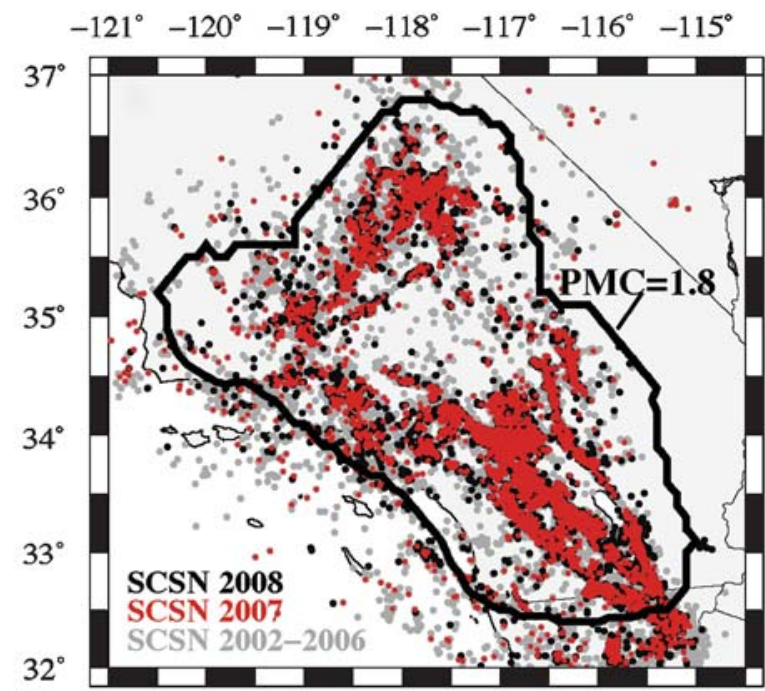

(b)

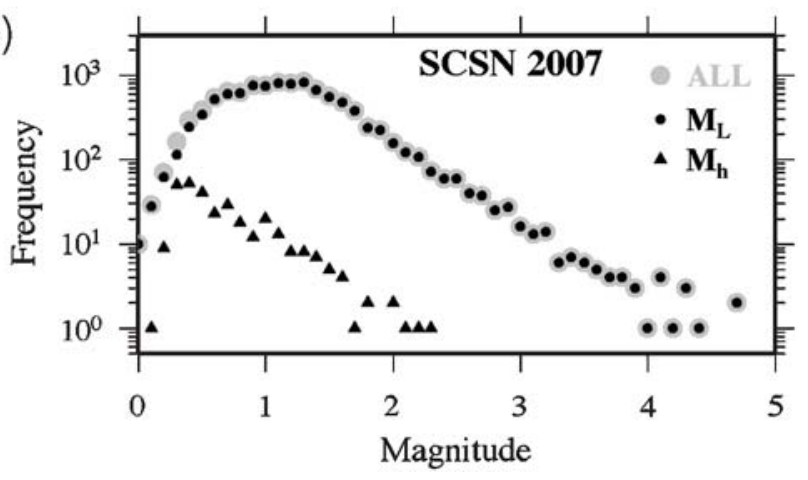

Figure 1. (a) Map of the study region in southern California. The polygon surrounds the area of $M 1.8$ completeness according to the PMC method at $99.9 \%$ probability. The gray dots represent all earthquakes between 1 January 2002 and 31 December 2006, the black dots represent the events that happened in 2008, while the red dots represent the earthquakes from 2007 for which both magnitudes are available. (b) Histogram of the contribution of both types of magnitude to each magnitude bin for the 2007 data. No $M_{\mathrm{w}}$ were observed in that year and within the polygon.

given as $M_{h}$. Those are hand-determined magnitudes, and in the current process of catalog recalibration, are being revised and wherever possible replaced by newly calculated $M_{\mathrm{L}}$ values. Therefore, the proportions change for the new cata$\log$ : for 2007 we find about $97 \%$ of the catalog given in $M_{\mathrm{L}}$ and only $3 \%$ in $M_{h}$. No $M_{\mathrm{w}}$ was observed in our study region in 2007. The regional distribution of the different magnitude types is perfectly even throughout the study region. Figure $1 \mathrm{~b}$ shows the histograms for 2007 of the contributions of the two magnitude types $M_{\mathrm{L}}$ and $M_{h}$ to each magnitude bin.

Figure 2 shows the changes in magnitudes for $M_{\mathrm{L}}$ and $M_{h}$ separately. Although the new magnitude calibration itself applies to $M_{\mathrm{L}}$ events only, the revision of $M_{h}$ events changes those magnitudes also (about half of them). For this search for a break in homogeneity, we therefore analyze the entire catalog, which will not only be the one predominantly used by researchers, but also allow us to estimate seismicity parameters such as the $b$-value, which is only sensibly defined on the set of all earthquakes, not those subsets of a certain magnitude definition.

For part of our rate change study, we use a declustered catalog from 2002 to 2008. Declustering, the separation between dependent and independent events, is a complex and controversial catalog analysis; its applicability depends on the purpose of each individual study (e.g., van Stiphout et al., 2010). However, for the purpose of identifying artifacts in reporting, declustering has been shown to be highly useful (e.g., Zuniga and Wiemer, 1999), revealing features that would otherwise be hidden in the numerous rate changes introduced by aftershock sequences and swarms. Several different approaches are available to remove aftershocks from earthquake catalogs. We decluster the catalog using the Reasenberg algorithm (Reasenberg, 1985) with standard parameters for California (summarized in Table 1). To obtain the declustered catalog, we use the data back to 1 January 1992 to account for the still large impact of the M 7.3 Landers earthquake in June 1992 on the ongoing seismic activity. But we use only the 1 January 2002 onward declustered data for this analysis. The declustered SCSN catalog includes 40,470 earthquakes for the six years and 5854 for 2007 alone.

\section{Analysis}

\section{Magnitude Differences}

The differences between the old and the new magnitudes for 2007 are shown in Figure 2a-c: for each event we plot the new versus the old magnitude and include via grayscale the frequency of observation. As explained previously, not only the $M_{\mathrm{L}}$ events feature new values, but also $M_{h}$. While some of the events are being corrected upward, most of the magnitude values are reduced. The differences become less striking for magnitudes above $M 2.3$, still even values above $M 4$ change slightly. The broad cloud of magnitude differences does not suggest a systematic change but a fluctuating signal, although the frequency of small reductions is highest. We found no geographical dependence of the differences. The largest differences between the old magnitude values and the new values reach a reduction of 2.3 units of magnitude and an increase of 1.5 units; the mean change is a reduction of the original magnitude by 0.13 units. For $4 \%$ of the $M_{\mathrm{L}}$ events, the magnitudes do not change with the new calibration. We find that $50 \%$ of the $M_{h}$ events change during the revision, with similarly scattering magnitude differences and a mean change of -0.21 units.

Figure $2 \mathrm{~d}-\mathrm{f}$ shows the changes in magnitude histograms between the old and the new magnitude data for 2007 for both magnitude types. Figure 3, which shows the annual magnitude histograms from 2002 to 2008, once for the declustered and once for the raw catalog, can provide a measure of how strong the changes seen in Figure $2 \mathrm{~d}-\mathrm{f}$ are in relation to annual scatter. Figure 3a demonstrates that the observed shift between the old and the new data for 2007 lies beyond 

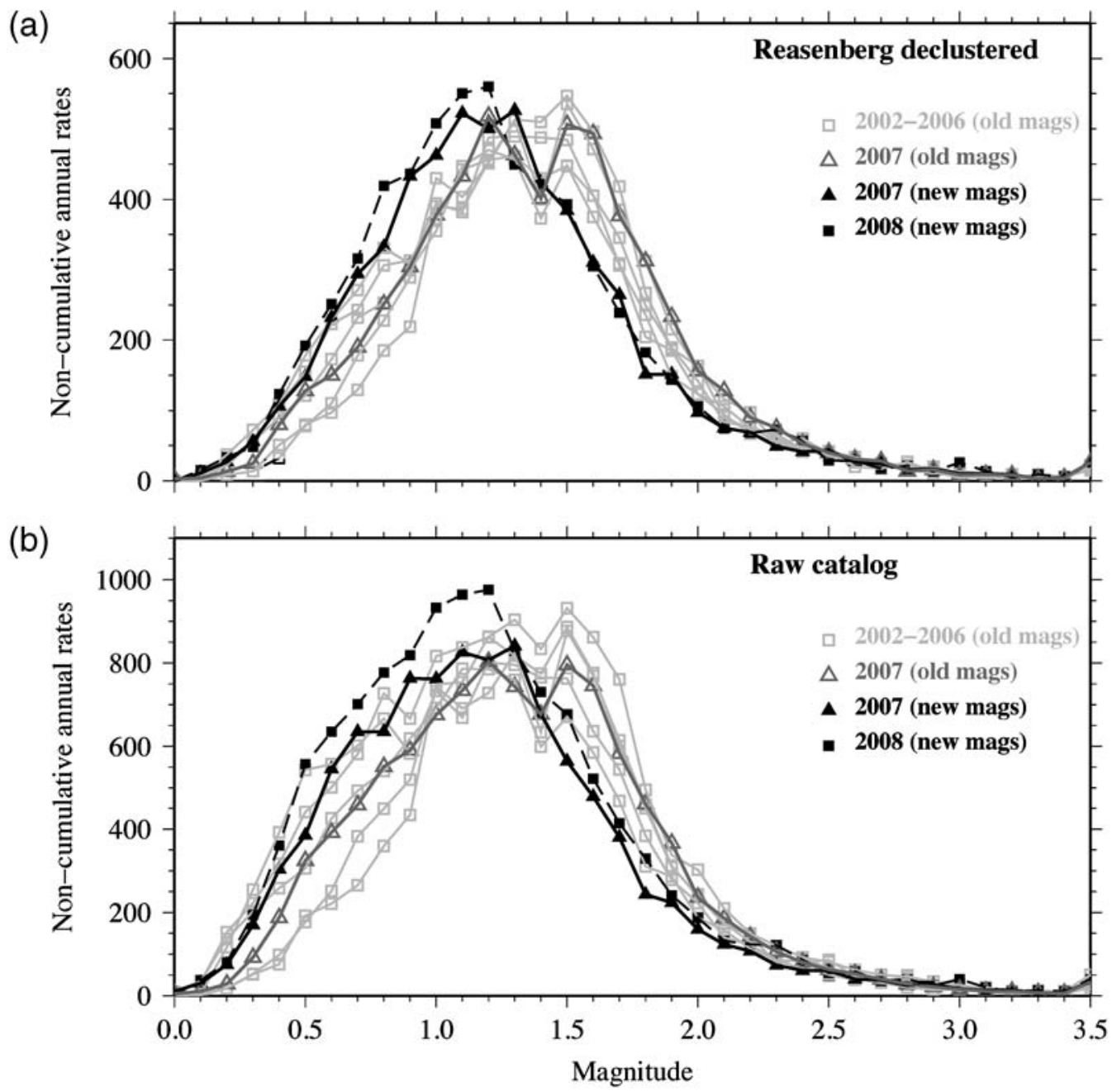

Figure 3. Annual magnitude histograms for (a) Reasenberg declustered catalog, and (b) raw catalog. The light gray represents data for old magnitudes from 2002 through 2006; dark gray, the old magnitude data for 2007; black, the new magnitude data: solid for 2007, dashed for 2008 .

or less stable number when looking at all events $M>0$ : 10,251 versus 10,142 in the new version of the catalog. That small difference of about $1 \%$ might be due to relocations of border events: some of them might not fall into our polygon any longer. When we cut the catalog at completeness magnitude $M \geq 1.8$, we observe a decrease in the number of events from 1865 in the old to 1267 in the new catalog, which is equivalent to a loss of $32 \%$. For the declustered catalog, the rate change reaches $33 \%$.

To measure the significance of the rate changes, we first calculate the percentage of change in the numbers of new versus old data for all magnitude cutoffs from $M 0$ to $M 4.0$ in 0.1 magnitude steps (i.e., all events $M 0$ and larger, $M 0.1$ and larger, etc.) (Fig. 4). For each magnitude cutoff, and therewith pair of observed numbers of earthquakes from the old and the new catalog, we calculate the Poisson probability that the change could have been observed by chance. The observed rate changes in Figure 4 are grayscale-coded by the confi- dence to which we can exclude a random occurrence based on their Poisson probability values. For the interpretation we distinguish between three classes of rate changes: those with $90 \%$ confidence, that is, probabilities of random occurrence of up to 10\%, which we call insignificant (light gray bars in the figure). They represent non-Poissonian behavior with no indication of an irregularity in the catalog. Confidence values between $90 \%$ and $99.99 \%$ are marginally significant (medium gray), and confidence values of more than $99.99 \%$ (dark gray) are statistically significant changes in the seismicity rate reporting (i.e., the probability that such an observation would have occurred by chance is less than $0.01 \%$ ).

We find highly significant rate decreases for cutoff magnitudes between $M 0.5$ and $M 2.2$, with the maximum decrease of $33 \%$ for $M$ 1.6. For cutoff magnitudes above $M 2.7$, we observe rate increases of up to $14 \%$. Because of the limited amount of data in those magnitude ranges, they turn out to be insignificant. 


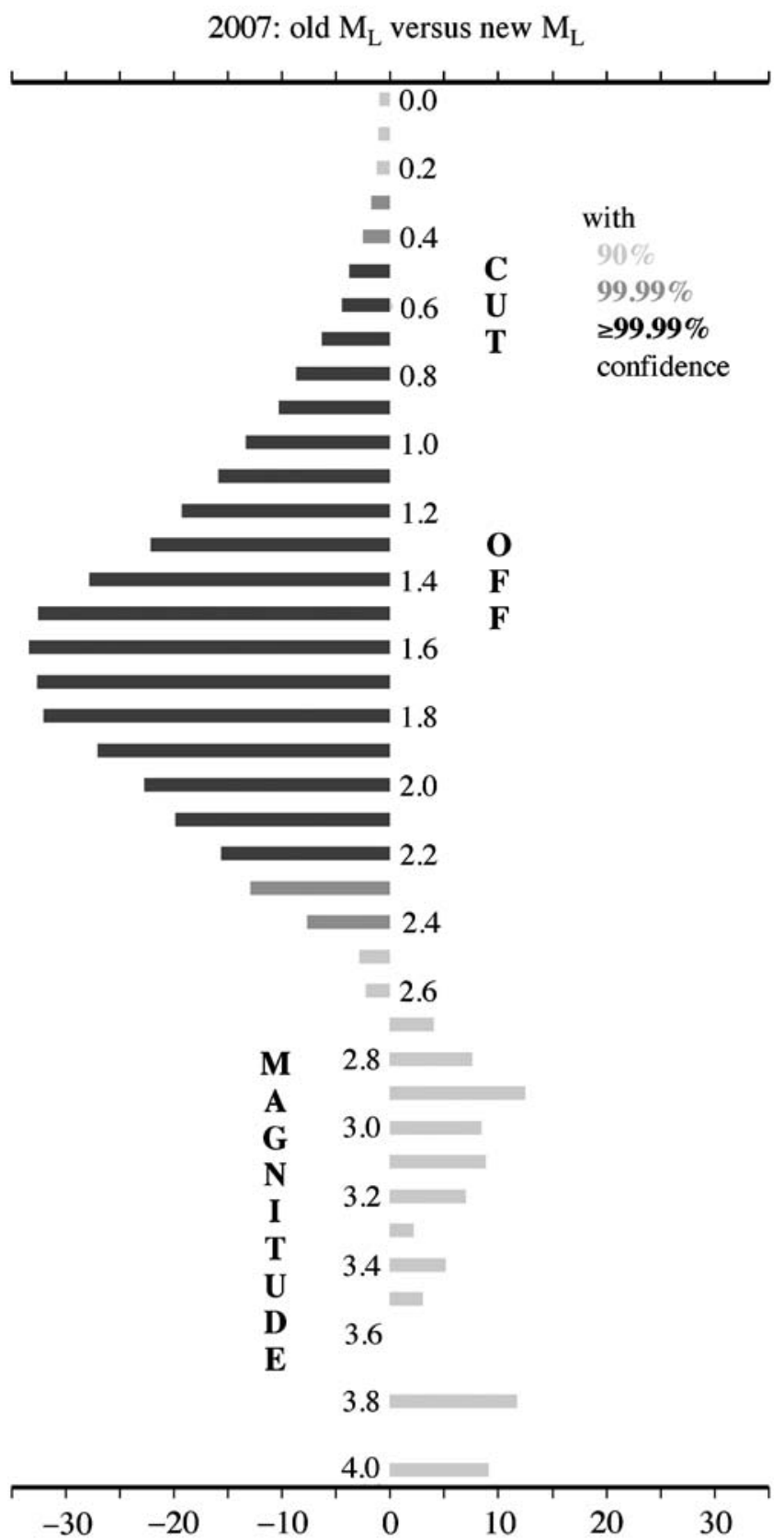

Observed rate change in $\%$

Figure 4. Observed rate changes between old and new data for 2007 and their significances for cutoff magnitudes $M 0.1$ to $M$ 4.0. Rate changes annotated in light gray are within $90 \%$ confidence limits and are insignificant, medium gray bars have significance values between $90 \%$ and $99.99 \%$, and rate changes in dark gray have statistically highly significant values with more than $99.99 \%$ confidence; the probability of an observation by chance is less than $0.01 \%$ for these changes.

\section{$M_{c}$ and $b$-Value Implications}

To give a reference for changes of the traditionally determined magnitude of completeness due to the magnitude recalibration, we calculate the EMR completeness values for the two catalogs for 2007 for all events $M>0$ within our polygon. We observe a drop from $1.6 \pm 0.02$ to $1.3 \pm 0.05$.

We calculate the $b$-values for both versions of the catalog for 2007 as well as a nearly continuous temporal evolution for 2002 through to 2008. Referring to the catalog independent probability-based magnitude of completeness definition, we assume $M 1.8$ to be the magnitude of completeness in the study region, so we use $M_{\text {min }}$ equals $1.8-0.05$ (where 0.05 corrects for the rounded magnitudes to 0.1 bins) and the data sets' mean magnitudes $M_{\text {mean }}$ to compute maximum-likelihood $b$-values by solving the equation (Utsu, 1965; Aki, 1965; Bender, 1983)

$$
b=1 /\left(M_{\text {mean }}-M_{\text {min }}\right) \times \log e .
$$

The confidence limit of this estimation is given by (Shi and Bolt, 1982)

$$
\sigma(b)=2.30 b^{2}\left(\sum_{i=0}^{n}\left(M_{i}-M_{\text {mean }}\right)^{2} / n(n-1)\right)^{1 / 2} .
$$

We find that the $b$-value for 2007 decreased by more than 0.2 units from $1.16 \pm 0.03$ for the old magnitude data to $0.95 \pm 0.03$ for the new magnitude data. To relate that change to the temporal fluctuation over the last few years (2002 to the end of 2008), we calculate $b$-values for a 600event window, moving it along with time, overlapping by 120 events with each step. Figure 5a shows the resulting evolution of $b$-values calculated from the two data sets with old and new magnitude definition. Data before 2007 are computed with the old magnitude calibration (black in Fig. 5a); for 2007 we have both values available, for 2008 onward only the new values (gray in Fig. 5a). The signal stands out clearly with the two curves diverging greatly. The mean $b$-value calculated from old magnitude data (2002-2007) is $1.1 \pm 0.01$; for the new magnitude data $(2007-2008)$ it is $0.94 \pm 0.02$.

Calculating the $b$-value as a function of cutoff magnitude allows a comparative evaluation of the two different magnitude scales. For a catalog that perfectly obeys the Gutenberg-Richter relationship (eq. 1), that is, above completeness is totally linear on a log scale, one would expect the $b$-value to increase from very small values for too low cutoff magnitudes and level out at the real value when approaching $M_{c}$. In the real world the $b$-value often changes again for higher magnitudes, depending on the shape of the cumulative frequency magnitude distribution, implying that the $b$-value function does not level out at the real value, but only forms a plateau. The characteristic of this plateau defines the stability and quality of the $b$-value and can be used to estimate the goodness of scaling in the catalog, which could be either naturally dominated or as in this case computationally. Figure $5 \mathrm{~b}$ shows the $b$-values for the two data sets (black: old magnitude calibration; gray: new magnitude calibration) obtained for different cutoff magnitudes. We require at least 50 events to calculate a $b$-value, which determines the cutoff magnitude range to be $M 0$ to $M 4.1$ for the old data (2002-2007) and $M 0$ to $M 3.7$ for the new magnitude data 
(a)

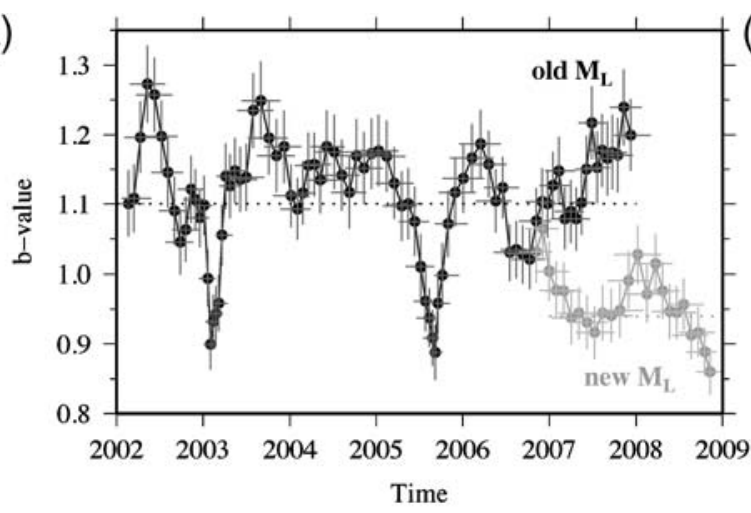

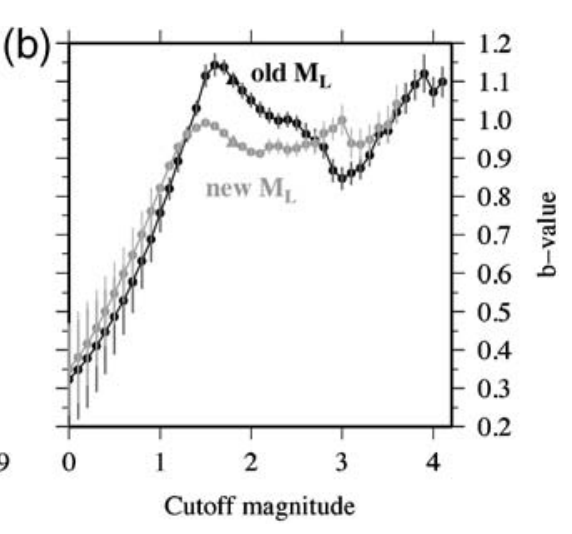

Figure 5. (a) Temporal evolution of $b$-values, calculated from 600 events (with 120 events overlap). We assume a completeness magnitude of $M$ 1.8. Horizontal bars represent the time interval used for each calculation, while vertical bars represent the confidence limits of $b$, following Shi and Bolt (1982). The black data are calculated from the old magnitudes, the gray data are based on the new calibration. Dotted lines show the mean $b$-values calculated for 2002-2007 and 2007-2008, respectively. (b) $b$-value as a function of cutoff magnitude, black symbols represent data with old magnitude calibration, gray symbols represent new data. The triangles mark the $b$-value that is used when assuming a completeness magnitude of $M 1.8$ as we do in this article. The error bars show the $b$-value range that is needed explain at least $50 \%$ of the data.

(2007-2008). The graph shows an unstable developing of $b$-values for the old magnitude data, which do not even form a plateau. The new magnitude data on the contrary feature a well-defined plateau, providing an improvement in $b$-value stability for the southern California catalog.

\section{Transformation}

For many applications it would be desirable if one had the option to transform the magnitudes of a sample from one magnitude scale into the other in order to perform analyses across the breakpoint in $M_{\mathrm{L}}$ calibration. Figure $2 \mathrm{~d}-\mathrm{f}$ shows the magnitude histograms for the two types of magnitude for the old and the new data of 2007. The histogram for the $M_{\mathrm{L}}$ data (Fig. 2e) suggests a shift toward lower magnitudes, which can be approximated by the mean change in magnitudes, -0.13 . Unfortunately, the nature of the changes previously discussed is such that a simple translation is impossible. The processing changes leading to the new magnitude distribution are complex, and, most importantly, not operating on the set of all earthquakes, but on each single event, with locally varying site corrections for the recording stations. Therefore, they cannot be properly represented by a mean correction function over the whole catalog. Only $96 \%$ of the $M_{\mathrm{L}}$ notated earthquakes are assigned a new magnitude, and the scatter of (a)

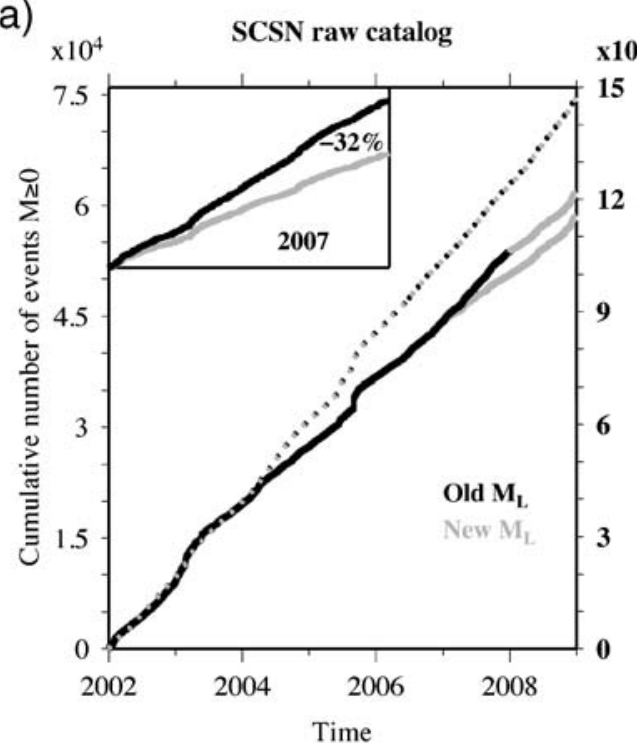

(b)

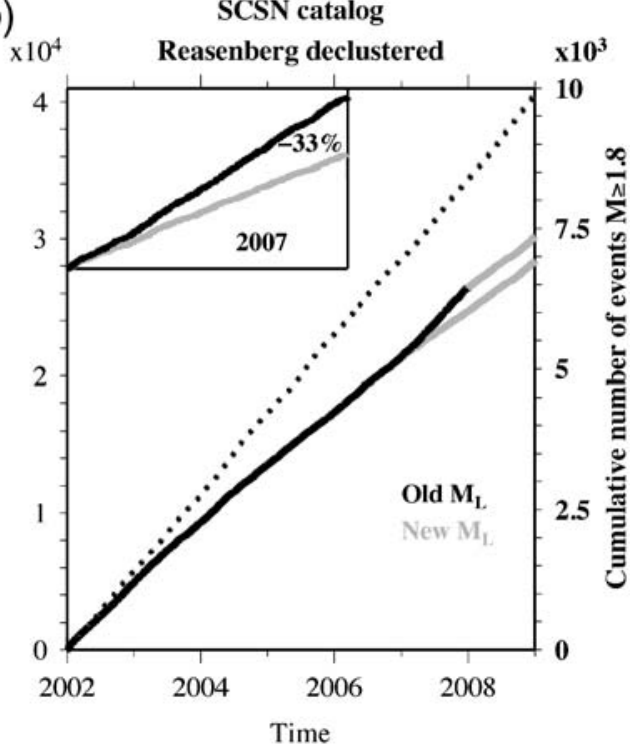

Figure 6. Cumulative number of events with time between January 2002 and December 2008; (a) shows the raw catalog, (b) is based on the Reasenberg declustered catalog. Black lines show catalog with old magnitude calibration, gray represents the new calibration. The dotted lines show the catalogs for $M \geq 0$. The catalogs are more or less the same in that case, so that the two lines are on top of each other and not distinguishable. The solid lines show the catalogs for $M \geq 1.8$. Those data are identical up to the end of 2006 , diverge by $32 \%$ in 2007 , and continue on parallel for 2008 . 
magnitude differences spans many multiples of the average change of -0.13 (Fig. 2a-c).

\section{Implications for Selected Applications}

There are two major fields of applications, which will predominantly be biased by the man-made rate and scaling changes documented in this article: rate change studies and hazard and risk assessments for southern California.

Changes in the rate of microseismicity are often related to changes in the stressing rate (e.g., Miller, 1996; Stein, 1999; Dieterich et al., 2000; Wyss and Wiemer, 2000; Toda and Stein, 2002) or dynamic triggering (Gomberg et al., 2001; Husen et al., 2004; Felzer and Brodsky, 2006). Precursory phenomena, such as precursory seismic quiescence, also require a very detailed analysis of the rate of small earthquakes (e.g., Wiemer and Wyss, 1994; Dieterich and Okubo, 1996; Joswig, 2001). For studies of this type, a regional rate change of more than $30 \%$ (see Fig. 6) poses a significant challenge that needs to be considered in the analysis. In the worst scenario, such changes introduced in the homogeneity of earthquake reporting may make it impossible to establish precursory signals with confidence. This was the case for the 2002 Denali $\left(M_{\mathrm{w}}\right.$ 7.9) earthquake (Ratchkovski et al., 2004). The precursory quiescence that is observed is highly suspect because the reporting homogeneity changed significantly in the years before.

Hazard forecasts are based on a variety of data from various fields of seismology and geology. One part of each hazard calculation is the computation of expected rates of events for all magnitude bins to estimate the Poissonian, or time-independent, background hazard. Many parameters go into hazard calculation algorithms, but here we note how lower $b$-values increase the rates for large events. Background hazard is computed from a declustered catalog, so we specifically calculate the annual rates for magnitude 2 , 3, 4, 5, and 6 events for the Reasenberg declustered catalog, contrasting the expected numbers based on the old $M_{\mathrm{L}}$ data (annual mean for 2002 to 2007), $b_{\text {old }}=1.11 \pm 0.01$ compared with the new $M_{\mathrm{L}}$ data (annual mean of 2007 and 2008), $b_{\text {new }}=0.92 \pm 0.02$ (Fig. 7). Although rates for large magnitude earthquakes cannot be reliably estimated on the basis of two years of a regional catalog, we show what would be calculated for magnitude 7 and 7.5 events based on the available data. We find that although the yearly rate $R$,

$$
R_{\text {new }}=\text { factor } \times R_{\text {old }},
$$

of $M 2$ events in the declustered catalog is more than $10 \%$ lower for the two years with the new magnitude data than for the six years with the old magnitude definition, the extrapolated rate for $M 7.5$ events is about 10 times higher using the $b$-value from the new data. Calculating annual rates for $M 6$ events, we expect five times more after the change of $M_{\mathrm{L}}$ calculation or vice versa; the rates have been underestimated by a factor of 5 because of inadequate $M_{\mathrm{L}}$ calculation before the recalibration. Indeed, the comparison of the expected rates with the annualized observed rates from the last 77 years of the Reasenberg declustered SCSN catalog proves that the new $b$-value with its extrapolated rates is representing the data better than the old one, but it still underestimates the real world $M \geq 6$ by a factor of about 2 . The $b$-value calculated from the $830 M \geq 4$ events between 1932 and 2008 is $0.87 \pm 0.03$. Table 2 summarizes the expected annual rates and changes (factors) for the Reasenberg declustered catalog as well as the annualized observed rates for $M \geq 4$ for the last 77 years of the SCSN catalog (the early catalog would not be complete
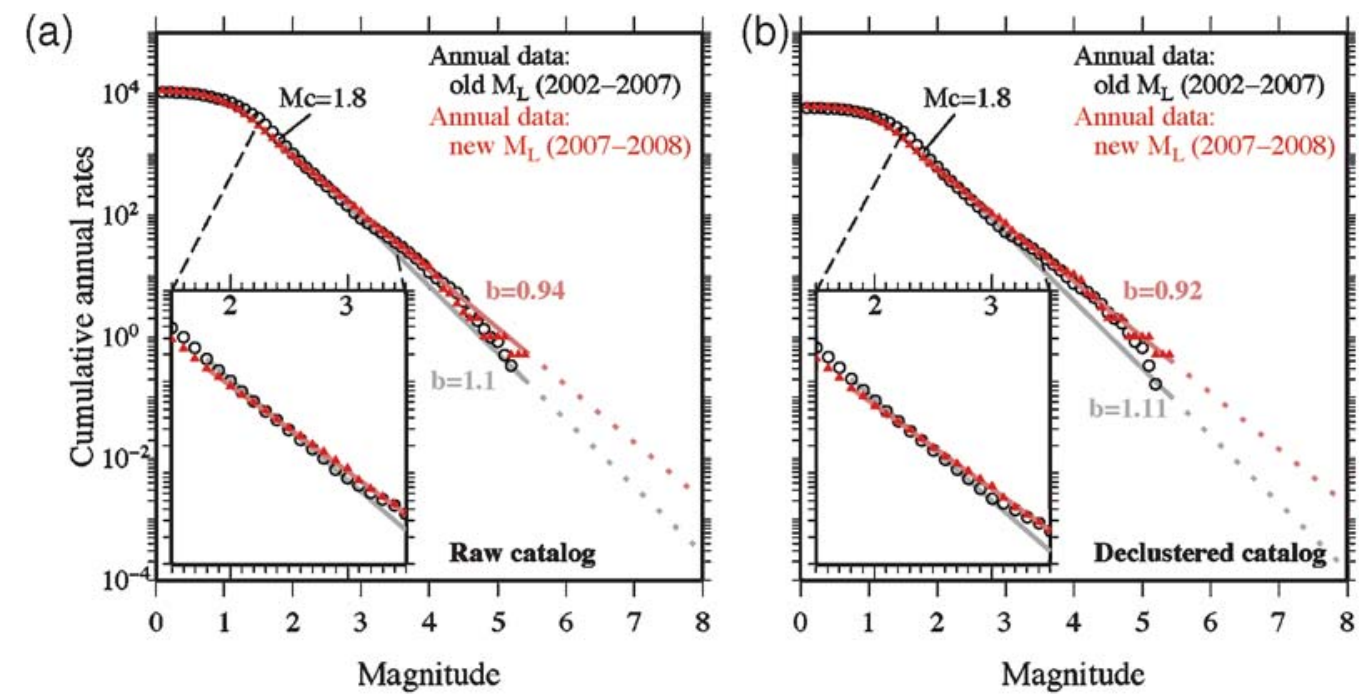

Figure 7. Annualized frequency magnitude distribution (FMD) for the old (black circles) and the new (red triangles) magnitude data. The zoom shows the differences in the slope between the curves for the small magnitudes above completeness, which dominates the calculation of the $b$-values. The different $b$-values (calculated with magnitude of completeness of $M 1.8$ ) are shown as gray and light red lines for the old and the new data, respectively. The dotted part of the $b$-value lines is the extrapolation toward large magnitudes, showing an increase of expected rates for large events of a factor of 10 and more. 
Table 2

Expected Annual Rates $R$ Calculated from the Different $b$-Values for Old $M_{\mathrm{L}}{ }^{*}$ and New $M_{\mathrm{L}} *$ for the Reasenberg Declustered Catalog ${ }^{\dagger}$

\begin{tabular}{lcccclll}
\hline Magnitude & 2 & 3 & 4 & 5 & \multicolumn{1}{c}{6} & \multicolumn{1}{c}{7} & \multicolumn{1}{c}{7.5} \\
\hline$R_{\text {old }}$ & 644.17 & 50.00 & 3.88 & 0.30 & 0.023 & 0.0018 & 0.0005 \\
$R_{\text {new }}$ & 561.69 & 67.53 & 8.12 & 0.98 & 0.12 & 0.014 & 0.0049 \\
Factor & 0.9 & 1.4 & 2.1 & 3.3 & 5.2 & 7.8 & 9.8 \\
$R_{\text {obs32-08 }}$ & - & - & 10.78 & 1.19 & 0.22 & 0.039 & 0.013 \\
\hline
\end{tabular}

*Old $M_{\mathrm{L}}$ is $b_{\text {old }}=1.11$; new $M_{\mathrm{L}}$ is $b_{\text {new }}=0.92$.

'Based on the annualized observed numbers of events above completeness magnitude $M 1.8,1074$ for 2002-2007 and 858 for 2007-2008.

Changes in the rates are expressed by factor $=R_{\text {old }} / R_{\text {new }}$. For comparison, we include the annualized numbers of observed events for $M \geq 4,5,6,7$, and 7.5 from the last 77 years of the Reasenberg declustered catalog (for that data set of 1083 events above $M 4$ in our study area, we calculate a $b$-value $b_{\mathrm{obs}}=0.87$ ).

for magnitudes 2 and 3) (Hutton et al., 2009). Although contemporary Probabilistic Seismic Hazard Assessment (PSHA) is based on more information than the extrapolated rates of small recent events (e.g., geologic slip rate on mapped faults, time since last historical event), our simple test shows that a significant impact on the PSHA for southern California is conceivable due to changes in computation of magnitude.

There are more applications that are affected by the change in magnitude calibration, for example, the probabilitybased magnitude of completeness method (PMC) (Schorlemmer and Woessner, 2008) whose results we used in this article. The method requires a sufficient period of homogeneous reporting before the time of interest. Therefore, with the documented break in homogeneity of reporting, which is being moved backward in time, the completeness maps will have to be continuously recalculated with respect to the new $M_{\mathrm{L}}$ calibration.

The change in $M_{\mathrm{L}}$ calculation also has the potential to bias the results for the CSEP testing center. Some models derive their parameters from data with local completeness values. For example, the Asperity-based Likelihood Model for California (Wiemer and Schorlemmer, 2007) estimates $a$ - and $b$-values locally using all earthquakes above completeness. Therefore, its parameter estimates and earthquake rate forecasts will differ with the new magnitude scale, although the target magnitudes for the actual test, constrained to $M \geq 4.5$, will likely be more or less unaffected by the rate changes. To optimize their prediction capabilities with the new magnitude scale, some of the forecasting models may need to be recalibrated.

\section{Discussion and Conclusion}

The new calibration for local magnitudes will provide an improved catalog. Still, the change in the homogeneity of reporting due to its introduction poses a serious challenge for many seismicity and seismic hazard related studies. The main purpose of this article is to make scientists aware of the change and to highlight some of the implications. As we have shown, seismicity rates and parameters change significantly between the catalog realizations for the two different magnitude scales; there is no simple translation from the old into the new scale. We therefore recommend, wherever possible, to restrict data to either the time before or after the change in $M_{\mathrm{L}}$ calculation. The second dimension of the challenge introduced by the recalibration is the reproducibility of analyses and results, a basic principle in science. The CISN continues to improve the catalog and plans to recompute local magnitudes for the SCSN catalog as far back as 1992. They started to recalculate $M_{\mathrm{L}}$ magnitudes in May 2009. The process, however, is slow and expected to take a few years to be finished back to 1992 . The current policy is a continuous updating of the catalog as soon as the new magnitudes become available month by month. Therefore, the break documented by us here for January 2007, will continuously move backward in time (and might change in amplitude, as the number of usable stations and therewith new station-corrections decrease for older data). This implies that the output of a catalog search does not any longer depend only on the search parameters like region, time window, and magnitude range, but also on the date on which it is downloaded. A thorough documentation of used data is inevitable to ensure reproducibility of the scientific research.

We have revealed in this article how strongly the change in $M_{\mathrm{L}}$ calibration affects the homogeneity of the southern California catalog and identified challenges for seismicity and implications for hazard related studies. We point out that there are good reasons for the SCSN to address these obstacles: the new magnitude scale will not only be uniform for all of California, which makes studies and results more comparable, but has also proven to better realize the Gutenberg-Richter relationship. $b$-values are important for a variety of applications, and a stable estimate is valuable.

Latest updated magnitudes at time of print: October 2005.

\section{Data and Resources}

We downloaded the SCSN catalog from the website www.data.scec.org/catalog_search/date_mag_loc.php. We used the link to the yearly .txt files for the current version 
of the catalog, while we downloaded the old data through the selection interface in early January 2009.

The magnitude of completeness data have been extracted from the website http://completeness.usc.edu.

Some of the analysis was done using the Matlab-based software package ZMAP (Wiemer, 2001), which can be downloaded from www.earthquake.ethz.ch/software/index.

The figures were created using the Generic Mapping Tools Version 4.2.1 (http://gmt.soest.hawaii.edu, Wessel and Smith, 1998).

\section{Acknowledgments}

We thank Jochen Woessner, Annemarie Christophersen, Kate Hutton, Max Wyss, Duncan Agnew, one anonymous reviewer, and the editorial board for valuable feedback that helped improve the manuscript. This research was supported by the Southern California Earthquake Center. SCEC is funded by the National Science Foundation Cooperative Agreement EAR-0106924 and USGS Cooperative Agreement 02HQAG0008. The SCEC contribution number for this article is 1408 .

\section{References}

Aki, K. (1965). Maximum likelihood estimate of $b$ in the formula $\log N=$ $a-b M$ and its confidence limits, Bull. Earthq. Res. Inst. 43, 237-239.

Bender, B. (1983). Maximum likelihood estimation of $b$-values for magnitude grouped data, Bull. Seismol. Soc. Am. 73, 831-851.

Dieterich, J., V. Cayol, and P. Okubo (2000). The use of earthquake rate changes as a stress meter at Kilauea volcano, Nature 408, 457-460.

Dieterich, J. H., and P. G. Okubo (1996). An unusual pattern of seismic quiescence at Kalapana, Hawaii, Geophys. Res. Lett. 23, 447-450.

Felzer, K. R., and E. E. Brodsky (2006). Decay of aftershock density with distance indicates triggering by dynamic stress, Nature 441, 735738.

Gomberg, J., P. Reasenberg, P. Bodin, and R. Harris (2001). Earthquake triggering by seismic waves following the Landers and Hector Mine earthquakes, Nature 411, 462-466.

Gutenberg, R., and C. F. Richter (1944). Frequency of earthquakes in California, Bull. Seismol. Soc. Am. 34, 185-188.

Habermann, R. E. (1987). Man-made changes of seismicity rates, Bull. Seismol. Soc. Am. 77, 141-159.

Habermann, R. E., and M. S. Craig (1988). Comparison of Berkeley and CALNET magnitude estimates as a means of evaluating temporal consistency of magnitudes in California, Bull. Seismol. Soc. Am. 78, 1255-1267.

Hellweg, M., R. Uhrhammer, K. Hutton, A. Walter, P. Lombard, and E. Hauksson (2007). Recalibrating $M_{\mathrm{L}}$ for the California Integrated Seismic Network, AGU Fall Meeting, S43A-1057, San Francisco.

Husen, S., S. Wiemer, and R. B. Smith (2004). Remotely triggered seismicity in the Yellowstone National Park region by the $2002 M_{\mathrm{w}}$ 7.9 Denali fault earthquake, Alaska, Bull. Seismol. Soc. Am. 94, S317-S331.

Hutton, K., J. Woessner, and E. Hauksson (2010). Earthquake monitoring in Southern California for 77 years (1932-2008), Bull. Seismol. Soc. Am. 100, 423-446.

Joswig, M. (2001). Mapping seismic quiescence in California, Bull. Seismol. Soc. Am. 91, 64-81.

Kanamori, H., P. Maechling, and E. Hauksson (1999). Continuous monitoring of ground-motion parameters, Bull. Seismol. Soc. Am. 89, 311-316.

Miller, S. A. (1996). Fluid-mediated influence of adjacent thrusting on the seismic cycle at Parkfield, Nature 382, 799-802.

Ratchkovski, N. A., S. Wiemer, and R. A. Hansen (2004). Seismotectonics of the central Denali fault, Alaska, and the 2002 Denali fault earthquake sequence, Bull. Seismol. Soc. Am. 94, S156-S174.

Reasenberg, P. A. (1985). Second-order moment of Central California Seismicity, J. Geophys. Res. 90, 5479-5495.
Richter, C. F. (1935). An instrumental earthquake magnitude scale, Bull. Seismol. Soc. Am. 25, 1-32.

Schorlemmer, D., and J. Woessner (2008). Probability of detecting an earthquake, Bull. Seismol. Soc. Am. 98, 2103-2117.

Schorlemmer, D., J. D. Zechar, M. J. Werner, E. H. Field, D. D. Jackson, T. H. Jordan , and RELM Working Group (2010). First results of the regional earthquake likelihood models experiment, Pure Appl. Geophys. 167, doi 10.1007/s00024-010-0081-5, in press.

Shi, Y., and B. A. Bolt (1982). The standard error of the magnitudefrequency $b$ value, Bull. Seismol. Soc. Am. 72, 1677-1687.

Stein, R. S. (1999). The role of stress transfer in earthquake occurrence Nature 402, 605-609.

Toda, S., and R. S. Stein (2002). Response of the San Andreas fault to the 1983 Coalinga-Nunez earthquakes: An application of interactionbased probabilities for Parkfield, J. Geophys. Res.-Solid Earth 107, art. no. 2126.

Utsu, T. (1965). A method for determining the value of $b$ in a formula $\log n=a-b M$ showing the magnitude frequency for earthquakes, Geophys. Bull. Hokkaido Univ. 13, 99-103.

van Stiphout, T., S. Wiemer, and D. Schorlemmer (2010). Uncertainties in seismicity rate estimation, J. Geophys. Res., in press.

Wiemer, S., and D. Schorlemmer (2007). ALM: An asperity-based likelihood model for California, Seism. Res. Lett. 78, 134-140.

Wiemer, S., and M. Wyss (1994). Seismic quiescence before the Landers $(M=7.5)$ and Big Bear $(M=6.5) 1992$ earthquakes, Bull. Seismol. Soc. Am. 84, 900-916.

Wiemer, S., and M. Wyss (2000). Minimum magnitude of complete reporting in earthquake catalogs: Examples from Alaska, the Western United States, and Japan, Bull. Seismol. Soc. Am. 90, 859-869.

Wiemer, S. S. (2001). A software package to analyze seismicity: ZMAP, Seism. Res. Lett. 72, 373-382.

Woessner, J., and S. Wiemer (2005). Assessing the quality of earthquake catalogues: Estimating the magnitude of completeness and its uncertainty, Bull. Seismol. Soc. Am. 95, 684-698.

Wyss, M., and S. Wiemer (2000). Change in the probability for earthquakes in Southern California due to the Landers magnitude 7.3 earthquake, Science 290, 1334-1338.

Zuniga, F. R., and S. Wiemer (1999). Seismicity patterns: Are they always related to natural causes?, Pure Appl. Geophys. 155, 713-726.

Swiss Seismological Service

Institute of Geophysics

ETH Zurich, NO H39.1

Sonneggstrasse 5

CH-8092 Zurich, Switzerland

Thessa.tormann@sed.ethz.ch

(T.T.)

Swiss Seismological Service

Institute of Geophysics

ETH Zurich, NO H57

Sonneggstrasse 5

CH-8092 Zurich, Switzerland

Stefan.wiemer@sed.ethz.ch (S.W.)

Seismological Laboratory

MS 252-21

California Institute of Technology

Pasadena, California 91125

Hauksson@gps.caltech.edu

(E.H.)

Manuscript received 25 May 2009 